\title{
34. On the Periods of Enriques Surfaces. I
}

\author{
By Eiji HoRIKAwA \\ University of Tokyo
}

(Communicated by Kunihiko KodaIRA, M. J. A., March 12, 1977)

§1. Introduction. A non-singular algebraic surface $S$ is called an Enriques surface if the following two conditions are satisfied:

(i) The geometric genus and the irregularity both vanish.

(ii) If $K$ is a canonical divisor on $S, 2 K$ is linearly equivalent to 0 .

Historically speaking Enriques surfaces were the first example of nonrational algebraic surfaces which satisfy the above condition (i). In this paper we are mainly interested in Enriques surfaces over the field of complex numbers $\boldsymbol{C}$.

From the condition (ii), it follows that there exists a two-sheeted unramified covering $\pi: T \rightarrow S$ such that $T$ is a $K 3$ surface. Since every $K 3$ surface is known to be simply-connected by Kodaira [6], $T$ is the universal covering of $S$. We take a holomorphic 2-form $\psi$ on $T$ which is non-zero everywhere, and consider the integrals

$$
\int_{r} \psi \quad \text { for } \gamma \in H_{2}(T, Z) \text {. }
$$

We let $\tau$ denote the covering transformation $T \rightarrow T$ over $S$ so that $\tau^{2}=$ id. Since $S$ has no holomorphic 2-form, we have $\tau^{*} \psi=-\psi$. On the other hand, $\tau$ acts on $H_{2}(T, Z)$ as an involution. If $\gamma$ is invariant by $\tau$, then the above integral (1) vanishes. Therefore the periods of $\psi$ are determined by the integrals (1) over those 2-cycles $\gamma$ satisfying $\tau \gamma=-\gamma$. Our main result is that the isomorphism class of $S$ is uniquely determined by these periods. A more precise statement will be given in $\S 4$. Details will be published elsewhere.

§2. Elliptic surfaces of index 2. It is known that an Enriques surface $S$ has a structure of an elliptic surface (see [1], [8]). That is, there exists a surjective holomorphic map $g: S \rightarrow \boldsymbol{P}^{1}$ whose general fibre $C$ is an elliptic curve. Moreover there exists a divisor $G$ on $S$ with $C G=2$. From Kodaira's formula for the canonical bundles of elliptic surfaces ([6], p. 772), it follows that $g$ has two multiple fibres, both being of multiplicity 2 . We view $S$ as an elliptic curve over the function field of $\boldsymbol{P}^{1}$. Then $G$ is a divisor of degree 2 on this curve. Hence $G$ defines a rational map $f_{1}$ of degree 2 of $S$ onto a rational ruled surface $W_{1}$. This map induces, for each generic fibre $C$, a double covering $C \rightarrow \boldsymbol{P}^{1}$ which is ramified at 4 points. Let $B_{1}$ be the branch 
locus of $f_{1}$. By applying elementary transformations defined in [3] we can modify $f_{1}$ into a similar rational map $f: S \rightarrow W$ onto a rational ruled surface $W$ such that its branch locus $B$ has only the following singularities :

(i) At most a simple triple point, that is, without infinitely near triple points (see [2]).

(ii) $B$ contains a fibre $\Gamma$ and $B_{0}=B-\Gamma$ has a double point $s$ on $\Gamma$ which, on performing a quadratic transformation at $s$, gives a double point of its proper transform on the proper transform of $\Gamma$.

A singularity of the second type corresponds to a double fibre. Hence in our case $B$ has exactly two of them. Here we remark that the choice of $W$ is not unique because, if we apply an elementary transformation at $s$ as in (ii), we obtain another ruled surface and the new branch locus still satisfies the above condition. Because of this phenomenon, we may always take $\boldsymbol{P}^{1} \times \boldsymbol{P}^{1}$ as $W$. In this way we obtain a birational model of $S$ which is a double covering of $\boldsymbol{P}^{1} \times \boldsymbol{P}^{1}$. This model is closely related to the model studied in [1] and [8], which is a double covering of $\boldsymbol{P}^{2}$.

$\S 3$. Two propositions. The construction in $\S 2$ proves the following

Proposition 1. Any two Enriques surfaces are deformation to each other.

To state the second proposition we recall that, if $T$ is a $K 3$ surface, $\mathrm{H}_{2}(T, Z)$ is an even unimodular euclidean lattice of signature $(3,19)$. Hence it is isomorphic to

$$
\Lambda=U_{1} \oplus U_{2} \oplus U_{3} \oplus E_{8} \oplus E_{8}^{\prime},
$$

where $U_{i}=Z x_{i}+Z y_{i}$ with $x_{i} y_{i}=1, x_{i}^{2}=y_{i}^{2}=0(i=1,2,3)$ and $E_{8}, E_{8}^{\prime}$ are copies of the unique even unimodular negative-definite lattice of rank 8. We define an involution $\rho: \Lambda \rightarrow \Lambda$ by the conditions $\rho \mid U_{1}=-i d$, $\rho\left(x_{2}\right)=x_{3}, \rho\left(y_{2}\right)=y_{3}, \rho\left(E_{8}\right)=E_{8}^{\prime}$ and that $\rho$ induces the identity $E_{8} \rightarrow E_{8}^{\prime}$. We fix $(\Lambda, \rho)$ once and for all.

Proposition 2. Let $T$ be the universal covering of an Enriques surface $S$. Then there exists an isomorphism of euclidean lattices $\varphi: H_{2}(T, Z) \rightarrow \Lambda$ which satisfies $\varphi \circ \tau=\rho \circ \varphi$, where $\tau$ denotes the involution on $H_{2}(T, Z)$ induced by the covering transformation.

By Proposition 1, it suffices to prove Proposition 2 for one special $S$. On the other hand, if $g: S \rightarrow P^{1}$ has a singular fibre of type II* (see Kodaira [5], p. 565), i.e., a singular fibre which has the configuration of the extended Dynkin diagram of type $\tilde{E}_{8}$, then it is easy to prove the proposition for $S$. Therefore the proof is reduced to constructing an Enriques surface with a singular fibre of type II*. This can be done by using the construction described in $\S 2$. 
$\S 4$. Main Theorem. $(\Lambda, \rho)$ being as above, we let $\Lambda(-1)$ denote the $(-1)$-eigenspace of $\rho$. Then $\Lambda(-1)$ is a euclidean lattice of signature $(2,10)$ with determinant $2^{10}$. Let $S$ be an Enriques surface and let $\varphi: H_{2}(T, Z) \rightarrow \Lambda$ be as in Proposition 2. Then the integrals (1) determines, via $\varphi$, a linear map $\omega: \Lambda(-1) \rightarrow C$ which satisfies the Riemann bilinear relation (see [7]). Hence $\omega$ can be viewed as a point of an open set $D$ in a quadric in $\boldsymbol{P}^{11}$. $D$ is a disjoint union of two copies of a 10-dimensional symmetric bounded domain of type IV. We let $\Gamma^{\prime}$ denote the group of those automorphisms of $\Lambda$ which commute with the involution $\rho$. Then $\Gamma^{\prime}$ induces a group $\Gamma$ of automorphism of $\Lambda(-1)$, and $\Gamma$ acts discontinuously on $D$. The image $\lambda(S)$ of $\omega$ on $D / \Gamma$ is uniquely determined by $S$ and does not depend on the choice of $\varphi$. We call $\lambda(S)$ the period of $S$.

Main Theorem. The isomorphism class of an Enriques surface $S$ is uniquely determined by its period $\lambda(S) \in D / \Gamma$.

The proof uses the Torelli theorem for $K 3$ surfaces due to PiateckiiShapiro and Shafarevich [7]. Suppose that two Enriques surfaces $S_{1}$ and $S_{2}$ have the same period. Then, if $\pi_{i}: T_{i} \rightarrow S_{i}(i=1,2)$ are the universal coverings, there exists an isomorphism $\varphi: H_{2}\left(T_{1}, Z\right) \rightarrow H_{2}\left(T_{2}, Z\right)$ which is compatible with involutions and preserves periods. If $\varphi$ maps effective cycles into effective cycles, then $\varphi$ is induced by a unique isomorphism $\Phi: T_{1} \rightarrow T_{2}$, and $\Phi$ is compatible with involutions. Hence $S_{1}$ and $S_{2}$ are isomorphic to each other. If $\varphi$ does not preserve effective cycles, then we can compose $\varphi$ with a reflexion

$$
\gamma \longrightarrow \gamma+\left(\gamma \cdot \pi_{2}^{*} e\right) \pi_{2}^{*} e
$$

with respect to the class $e$ of a rational curve on $S_{2}$. Composing $\varphi$ with a finite number of such reflexions we may assume that either $\varphi$ or $-\varphi$ preserves effective cycles, and then we are through as above.

Remarks, 1) From the explicit description of $(\Lambda, \rho)$, it follows that $D / \Gamma$ is connected.

2) It can also be proved that $\Gamma$ is an arithmetic subgroup of $S O(2,10)$ with respect to the $Q$-structure defined by $\Lambda(-1)$.

3) It is very likely that our method in [4] can be applied to study the image of the period map $\lambda$ for Enriques surfaces.

4) Our construction in $\S 2$ also works over any algebraically closed field of characteristic $\neq 2$. Using this construction we can prove that any Enriques surface in characteristic $\neq 2$ can be lifted to characteristic 0 .

\section{References}

[1] Artin, M.: On Enriques' surfaces. Ph. D. thesis, Harvard Univ. (1960).

[2] Horikawa, E.: Algebraic surfaces of general type with small $c_{1}^{2}$, I. Ann. 
of Math., 104, 357-387 (1976).

[3] - - On algebraic surfaces with pencils of genus 2. Iwanami Shoten and Cambridge Univ. Press (to appear in "Complex Analysis and Algebraic Geometry", a volume dedicated to K. Kodaira).

[4] - : Surjectivity of the period map of $K 3$ surfaces of degree 2 (to appear).

[5] Kodaira, K.: On compact complex analytic surfaces II. Ann. of Math., 77, 563-626 (1963).

[6] - : On the structure of compact complex analytic surfaces I. Amer. J. Math., 86, 751-798 (1964).

[7] Piateckii-Shapiro, I. I., and Shafarevich, I. R.: A Torelli theorem for algebraic surfaces of type K3. Izv. Akad. Nauk USSR, 35, 530-572 (1971).

[8] Shafarevich, I. R., et al.: Algebraic surfaces. Proc. Steklov Inst., 75 (1965) ; English translation. Amer. Math. Soc. (1967). 